\title{
73P/Schwassmann-Wachmann 3-B observed from the optical ground station
}

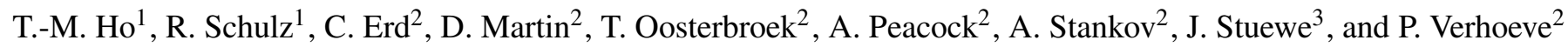 \\ ${ }^{1}$ Research and Scientific Support Department of ESA, ESTEC, Keplerlaan 1, 2200 AG Noordwijk, The Netherlands \\ e-mail: tra-mi.ho@esa.int \\ 2 Science Payload and Advanced Concepts Office of ESA, ESTEC, Keplerlaan 1, 2200 AG Noordwijk, The Netherlands \\ 3 Sterrewacht Leiden, Nils Bohrweg 2, 2333 CA Leiden, The Netherlands
}

Received 8 February 2007 / Accepted 17 September 2007

\begin{abstract}
Aims. In 2006 comet 73P/Schwassmann-Wachmann 3, which split in 1995 into five pieces, approached the Sun again with a swarm of new fragments. The same year in May, the conglomerate of sub-fragments from the original fragment B was observed with the SCam3 instrument mounted on the 1-m ESA Optical Ground Station (OGS) telescope in Tenerife, Spain. With a total FOV of $\sim 876 \mathrm{~km}$ $\times 730 \mathrm{~km}$ and a spatial resolution of $\sim 73 \mathrm{~km} /$ pixel, the $\mathrm{S}-\mathrm{Cam} 3$ observations provided the possibility to examine dust fragmentation processes, as well as dust and gas outflow, within the first few hundred kilometres of the sub-fragment surfaces.

Methods. The superconducting camera, S-Cam3, is an ultra-fast photon counting camera developed by ESA. Cooled to $\sim 0.3 \mathrm{~K}$, its sensitive superconducting tunnel junction sensors detect single photons, measuring their arrival time to accuracies of microseconds and determining its crude wavelength. The camera is also essentially noise-free except for sky background photons. Thus S-Cam3 essentially provides high-speed, low-resolution spectra between $395 \mathrm{~nm}$ and $1052 \mathrm{~nm}$ with a resolution of $\sim 35 \mathrm{~nm}$ at $500 \mathrm{~nm}$ wavelength.

Results. The images acquired show three intensity maxima that were identified as most likely from the B fragment itself and two clusters of sub-fragments $253 \mathrm{~km}$ and $896 \mathrm{~km}$ away from fragment B. Furthermore we could see spatial intensity variations on short time scales (2-4 min), indicating the varying dust and gas emission of "subnuclei". The gas and dust profiles do not show an inverse radial distribution $(1 / r)$ in all flow directions, but rather a clear deviation from a free radial outflow. This most likely is due to the gas outflow of one cluster of sub-fragments hitting the outflow of the other cluster. In other words, the material is expanding from one cluster into the other. In addition, the dust particles continue to fragment.
\end{abstract}

Key words. comets: individual: 73P-Schwassmann-Wachmann 3-B - instrumentation: detectors - methods: data analysis techniques: photometric - techniques: spectroscopic

\section{Introduction}

During a photographic search for asteroids A. Schwassmann and A. A. Wachmann discovered comet $73 \mathrm{P} /$ SchwassmannWachmann 3 (73P/SW 3) as it passed within 9.3 million kilometres of the Earth in 1930. With a relatively short orbital period of 5.4 years, this comet is a frequent visitor to the inner Solar System, but it was the last three returns that made it so interesting. In 1995, the comet nucleus split into five pieces (Fragments A to E) after it had undergone a major outburst. The outburst was detected in $\mathrm{OH}$ by radio observations and increased the nucleus activity by a factor of 20 (Crovisier et al. 1996). From the visual brightness of the central coma condensation in 1930 (Baldet 1930a,b), the radius of the comet nucleus before break-up was estimated to be $1.2 \mathrm{~km}$ (Sekanina et al. 1998). In December 1994, Boehnhardt et al. (1999) determined a radius of $1.1 \mathrm{~km}$ from observations at a large heliocentric distance. However as the comet already showed a weak coma and tail, the $1.1 \mathrm{~km}$ radius should be regarded as an upper limit for the size of $73 \mathrm{P} / \mathrm{SW}$.

Of the five fragments created in 1995, only three (B, C, E) were still visible at the comet's 2001 return. But when 73P/SW approached the Sun again in 2006, seven fragments were initially observed and more were discovered subsequently, indicating that the comet was breaking apart again.
From narrowband photometry, gas and dust production rates of $\log Q\left[\mathrm{H}_{2} \mathrm{O}\right]=27.59 ; \log Q[\mathrm{CN}]=25.06 ; \log Q\left[\mathrm{C}_{3}\right]=23.35 ;$ $\log Q\left[\mathrm{C}_{2}\right]=24.24$; $\log \operatorname{Af} \rho=1.5$ were determined for fragment B on 25 April 2006, a heliocentric distance of $1.65 \mathrm{AU}$ (Schleicher et al. 2006).

When the comet was observed by the Hubble Space Telescope (HST) on 28 April 2006, over 33 separate fragments were detected stretching across several degrees on the sky. Ground-based observers noted strong brightening events associated with some of the fragments, indicating that they were continuing to break up and that some may have disappeared altogether. HST tracked the two fragments, B and G, shortly after major activity outbursts. These observations revealed an extensive process of destruction in which the larger fragments were continuing to break up into smaller chunks. Thus several dozens of "mini-fragments" were trailing behind the main fragment (Weaver et al. 2006). The HST images of the B fragment, taken a few days apart, suggest that these chunks were pushed down the tail by outgassing from the icy, sunward-facing surfaces of the chunks. Smaller chunks were accelerated away from the parent fragment faster than the larger ones, indicating that their size was correlated to their mass. Some of the chunks seemed to dissipate completely over the course of several days.

The continuing disintegration of the B fragment of Comet 73P/SW 3 was again observed on 6 May 2006 with the ultra-fast 
Table 1. Observing geometry for 6 May 2006.

\begin{tabular}{ll}
\hline \hline Parameter & \\
\hline heliocentric distance [AU] & 1.054 \\
geocentric distance [AU] & 0.096 \\
Phase angle [deg] & 59.88 \\
\hline
\end{tabular}

photon counting camera, S-Cam3, developed by ESA. It consists of an array of superconducting tunnel junction detectors, working at a temperature of $0.3 \mathrm{~K}$, and it allowed the detection of fast changes during the fragmentation of the comet. For details of the instrument, see Martin et al. (2006) and references therein. The observations allowed the derivation of intensity and colour maps, as well as analysis of brightness variations on very short time scales. In addition the outflow dynamics could be examined.

\section{Instrumentation, observation, and data reduction}

Disintegrating comet 73P/Schwassmann-Wachmann 3 was observed with S-Cam3 at the ESA Optical Ground Station (OGS) 1-m telescope at Teide Observatory, Tenerife on 6 May 2006. S-Cam3 is a photon-counting detector consisting of a $12 \times 10$ array of Ta/Al Superconducting Tunnel Junctions cooled with liquid helium to $\sim 0.3 \mathrm{~K}$ (Martin et al. 2006). Each junction has a size of $35.5 \times 35.5 \mu \mathrm{m}^{2}$ with inter-pixel gaps of $<2 \mu \mathrm{m}$. The four corner pixels are not operated to obtain overall stability in the instrument. The total FOV is $12^{\prime \prime} \times 10^{\prime \prime}$, making the pixel size equivalent to $1^{\prime \prime}$, which resulted in a spatial resolution of $\sim 73 \mathrm{~km} /$ pixel at the comet owing to its very small geocentric distance (0.096 AU). Consequently, the FOV of S-Cam3 was $876 \mathrm{~km} \times 730 \mathrm{~km}$. The observation geometry is provided in Table 1.

The S-Cam3 instrument counts the number of incoming photons with a time resolution of a few microseconds and determines the energy of each incoming photon. It provides a bipolar response signal to a photon arrival event: a positive peak followed by a zero crossing and a negative peak. The amplitude of the bipolar pulse (PHA) is converted to photon energy (using calibration data for individual pixels) and the zero crossing into photon arrival time. The conversion between PHA and energy is described by

PHA = Energy $(e V) \times$ gain + offset,

whereby gain $=1328.05$ and offset $=34.75$. These values are obtained by calibration observations throughout each campaign. Afterwards the photon wavelength can be determined from its energy $(\mathrm{eV})$. For S-Cam3, photons have been selected with $\lambda$ between $395 \mathrm{~nm}$ and $1052 \mathrm{~nm}$. The spectral resolution of the instrument is $\sim 35 \mathrm{~nm}$ at $500 \mathrm{~nm}$ wavelength. Figure 1 gives the total number of photons per pixel for a time interval of $100 \mathrm{~s}$ within two selected regions of the comet, which are indicated as boxes in Fig. 2.

The reduction process covers flat-fielding and sky background subtraction. We also corrected the images for extinction by applying the atmospheric extinction curve, $k_{\lambda}$, as a function of wavelength, $\lambda$, derived from extensive measurements covering many years at La Palma (King 1985; La Palma Technical Note 31). Finally the images were absolutely calibrated with a Solar Analog star since no Standard Stars measurements were obtained for this campaign. Because of the limited knowledge of the consensus of the spectra of the Solar Analog star and the Sun, a $30 \%$ uncertainty is obtained in absolute terms for the blue
Upper Box

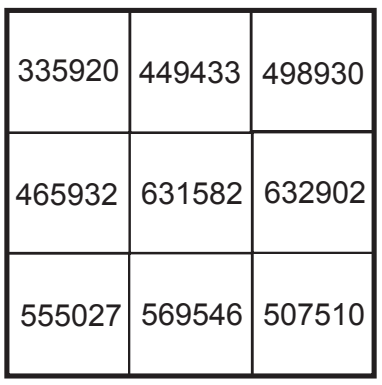

Lower Box

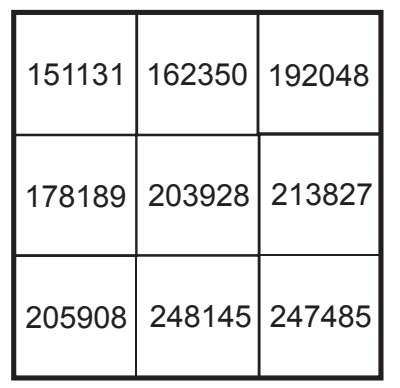

Fig. 1. The total photons per pixel for an integration time of $100 \mathrm{~s}$ of two selected regions indicated as upper and lower boxes in Fig. 2.

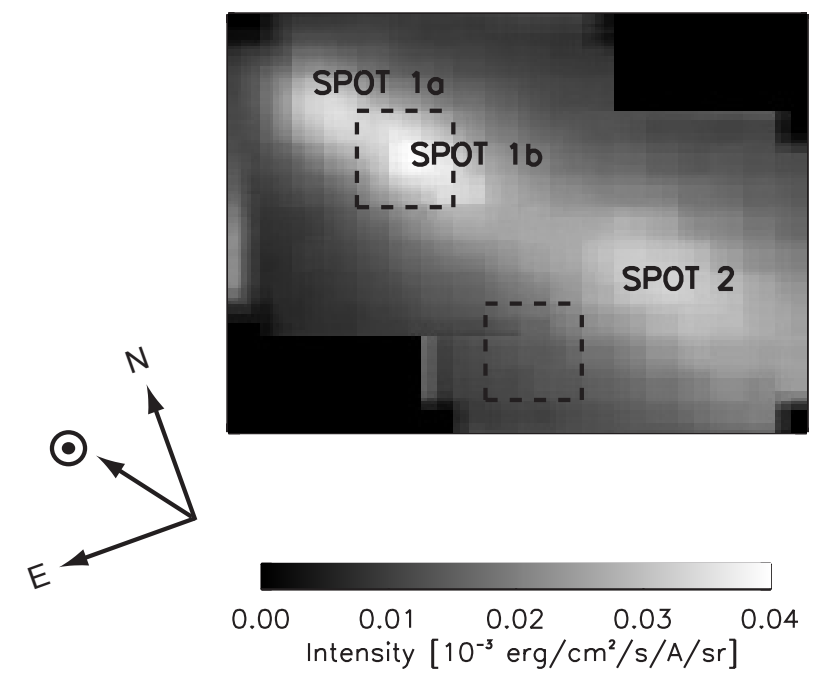

Fig. 2. Composite image of Spot $1 \mathrm{a}, \mathrm{b}$ and Spot 2 of $73 \mathrm{P} / \mathrm{SW} 3 \mathrm{~B}$. The image has been oversampled by a factor of 10 and smoothed by 5 pixels. The total FOV of this image is $1314 \mathrm{~km} \times 949 \mathrm{~km}$. The direction of the Sun, North, and East are indicated. The upper and lower boxes (dashed lines) mark the pixel arrays presented in Fig. 1.

narrow band. Thus, any conclusion should be derived from the relative colours.

Photon-counting observations of comet $73 \mathrm{P} / \mathrm{SW} 3 \mathrm{~B}$ were taken from 3:36 UT to 4:46 UT. Because of the limited FOV of the instrument, we performed a non-sidereal tracking of the distinct regions of the comet separately with the nominal differential velocities that were produced by the JPL ephemeris. The rate of changes are presented in Table 2 . We used relative off-set pointing to adjust the telescope to view the various spots.

The total data set is summarised in Table 2 where the nomenclature refers to specific regions (edge, Spot1, and Spot2) of $73 \mathrm{P} / \mathrm{SW} 3 \mathrm{~B}$ that were observed. Note that each image in Table 2 can be divided into many images with shorter photon integration times, whereby these times can be selected from within the data set, as needed, with the minimum integration time being only limited by the signal strength and therefore by the Poisson photon-counting statistics. Since the camera is photon-counting, these integration times are only a feature of the post observation data analysis and not related to hardware configurations.

\section{Data analysis}

Figure 2 shows the composite of several images of the $100 \mathrm{~s}$ counting time, comprising Spot 1 and Spot 2 (note Spot 1 actually consists of two intensity maxima named Spot1a and Spot1b). 
Table 2. List of observations.

\begin{tabular}{lccccc}
\hline \hline Image & Date & UT-Start & Counting Time [s] & $\mathrm{d}(\mathrm{RA}) / \mathrm{d} t\left[^{\prime \prime} / \mathrm{h}\right]$ & $\mathrm{d}(\mathrm{Dec}) / \mathrm{d} t\left[^{\prime \prime} / \mathrm{h}\right]$ \\
\hline edge_0001 & $2006 / 05 / 06$ & $03: 26: 22$ & 77 & 392.24 & 110.75 \\
spot_1_0001 & $2006 / 05 / 06$ & $03: 32: 06$ & 114 & 392.64 & 110.35 \\
spot_1_0002 & $2006 / 05 / 06$ & $03: 35: 15$ & 115 & 392.75 & 110.14 \\
spot_1_0003 & $2006 / 05 / 06$ & $03: 58: 45$ & 100 & 393.74 & 108.52 \\
spot_1_0004 & $2006 / 05 / 06$ & $04: 00: 30$ & 100 & 393.83 & 108.38 \\
spot_1_0005 & $2006 / 05 / 06$ & $04: 02: 16$ & 100 & 393.88 & 108.31 \\
spot_1_0006 & $2006 / 05 / 06$ & $04: 04: 01$ & 100 & 393.96 & 108.18 \\
spot_1_0007 & $2006 / 05 / 06$ & $04: 05: 46$ & 100 & 394.06 & 108.04 \\
spot_1_0008 & $2006 / 05 / 06$ & $04: 11: 32$ & 100 & 394.34 & 107.64 \\
spot_1_0009 & $2006 / 05 / 06$ & $04: 13: 17$ & 100 & 394.40 & 107.57 \\
spot_1_0010 & $2006 / 05 / 06$ & $04: 15: 03$ & 100 & 394.50 & 107.44 \\
spot_1_0011 & $2006 / 05 / 06$ & $04: 16: 48$ & 100 & 394.59 & 107.31 \\
spot_1_0012 & $2006 / 05 / 06$ & $04: 18: 33$ & 100 & 394.70 & 107.17 \\
spot_1_0013 & $2006 / 05 / 06$ & $04: 20: 18$ & 100 & 394.75 & 107.11 \\
spot_1_0014 & $2006 / 05 / 06$ & $04: 22: 03$ & 100 & 394.85 & 106.97 \\
spot_1_0015 & $2006 / 05 / 06$ & $04: 23: 48$ & 100 & 394.90 & 106.91 \\
spot_1_0016 & $2006 / 05 / 06$ & $04: 25: 34$ & 100 & 395.06 & 106.71 \\
spot_2_0001 & $2006 / 05 / 06$ & $04: 30: 55$ & 100 & 395.34 & 106.37 \\
spot_2_0002 & $2006 / 05 / 06$ & $04: 32: 40$ & 100 & 395.44 & 106.24 \\
spot_2_0003 & $2006 / 05 / 06$ & $04: 34: 26$ & 100 & 395.50 & 106.18 \\
spot_2_0004 & $2006 / 05 / 06$ & $04: 36: 11$ & 150 & 395.52 & 106.04 \\
spot_2_0005 & $2006 / 05 / 06$ & $04: 38: 46$ & 100 & 395.78 & 105.85 \\
spot_2_0006 & $2006 / 05 / 06$ & $04: 40: 31$ & 100 & 395.90 & 105.72 \\
spot_2_0007 & $2006 / 05 / 06$ & $04: 42: 17$ & 100 & 396.00 & 105.65 \\
spot_2_0008 & $2006 / 05 / 06$ & $04: 44: 02$ & 100 & 396.07 & 105.52 \\
spot_2_0009 & $2006 / 05 / 06$ & $04: 45: 47$ & 100 & 296.16 & 105.39 \\
\hline & & & & & \\
\hline & & & &
\end{tabular}

To optimise the signal resolution, the images have been shifted so that their centres overlap (see Sect. 3.1 for details) and then stacked. According to the Sekanina model, which describes the disintegration dynamics through five parameters - the splitting time $T_{\mathrm{s}}$, the radial velocity $V_{\mathrm{r}}$, transverse velocity $V_{\mathrm{t}}$, and normal components $V_{\mathrm{n}}$ of the separation velocity of the secondary fragment relative to the primary one, and deceleration $\Gamma$, under the assumption that $73 \mathrm{P} / \mathrm{SW} 3$ did not break up by tidal forces - the fragment configuration of this split comet should be such that the main body is leading with all the companions trailing behind (Sekanina 1982, 1999). Based on this we conclude that Spot1a most likely contains the main B fragment, while Spot1b and Spot 2 are two clusters of "mini-comets" (=sub-nuclei) that are a few hundred metres in size so unfortunately cannot be resolved.

For the detailed analysis, we over-sampled all images by a factor of 10 to form an array of $120 \times 100$ pixels and applied a smoothing function (boxcar average) on these images for better visualization, if required e.g. in the case of investigating profiles. Oversampling was applied to consider an intensity variation of $1 / 2$ pixel. Any variations of less than $1 / 2$ of the real pixel ( $\equiv 5$ pixel of the resized image) are excluded in the data interpretation. Smoothing over 5 pixels was applied and its validity tested using the $\chi^{2}$-goodness-of-fit. It showed that the smoothing of Spot 2 resulted in a good representation of the unsmoothed images, while the smoothed images of Spot1 did not perfectly fit the original ones in all cases in the region of the gap between Spot $1 \mathrm{a}$ and $1 \mathrm{~b}$ at $450-500 \mathrm{~km}$ (see Fig. 2). The main reason for this is that the gap between the two maxima is less than one pixel wide, which makes the over-sampling very sensitive to seeing variations and tracking errors. Nevertheless, this point notwithstanding, a smoothed image for the data evaluation was used in both cases to facilitate an improved recognition when needed.

All three spots have an elliptical shape. To estimate their sizes and their contribution to the total gas and dust production of

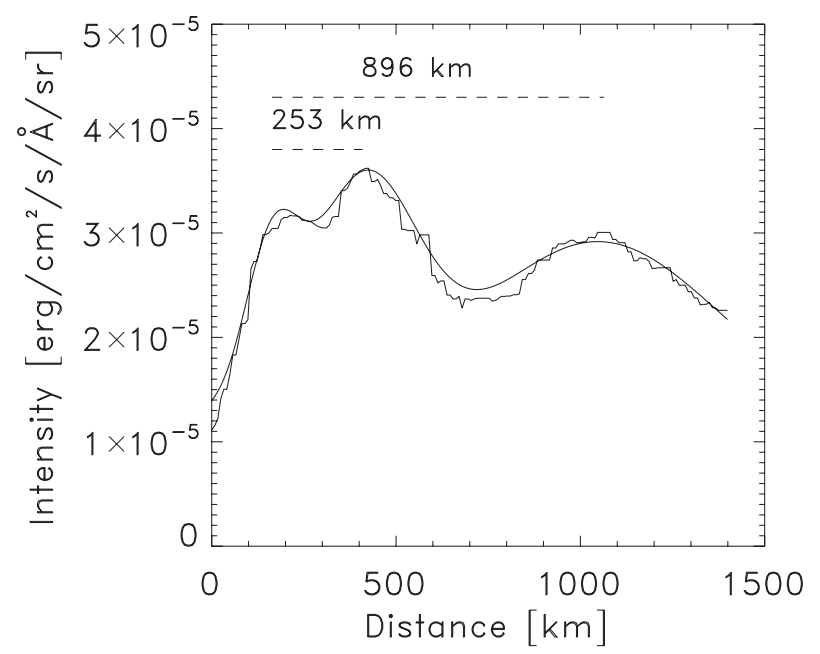

Fig. 3. A three Gaussian fit to the sun- to tailward profile of Spot1 and Spot2. The distances of Spot $1 b$ and Spot 2 in respect to the main B fragment $(=$ Spot 1a) are indicated.

fragment $\mathrm{B}$, we approximated the intensity profiles of the three spots by two-dimensional Gauss functions. Three Gaussians were fitted to the sun-tail profile and to the perpendicular profiles of Spot1a, 1b, and Spot 2 to obtain the dimensions along the long and short axes. The fitted sun-tail profile is shown in Fig. 3 and Table 3 summarises the results of the fits. The dimensions of the spots in full width half maximum $(F W H M)$ have been corrected for a seeing of $1.9^{\prime \prime} F W H M$, i.e. we have deconvolved the observed dimensions. It clearly shows that Spotla has the smallest extension and lowest intensity of all three spots, although it should contain the main B fragment.

Spot $1 b$ is about twice the extent of Spot1a, and it contributes a factor of three more to the total intensity. It probably contains many sub-nuclei that were formed by further disintegration 
Table 3. The results of the fit to the profiles of Spot1 and Spot2.

\begin{tabular}{lccc}
\hline \hline Emission & $\begin{array}{c}1-F W H M^{1} \\
{[\mathrm{~km}]}\end{array}$ & $\begin{array}{c}s-F W H M^{1} \\
{[\mathrm{~km}]}\end{array}$ & $\begin{array}{c}\text { Contribution to total } \\
\text { fragments' signals [\%] }\end{array}$ \\
\hline Spot1a & 131 & 134 & 9 \\
Spot1b & 302 & 177 & 21 \\
Spot2 & 825 & 334 & 71 \\
\hline
\end{tabular}

${ }^{1} 1-F W H M$ describes the dimension of the long-axis along the Suntailward direction and s-FWHM describes the dimension of the short-axis.

of bigger fragments ("secondaries", "tertiaries"), which are spatially wider distributed because of the different separation velocity vectors of the fragments. The presence of active sub-nuclei in Spot 1b compared to that of Spotla also explains the higher contribution of Spot $1 b$ to the total intensity. Note that the process of multiple fragmentations has been investigated by Sekanina et al. (1998) and is summarised by Boehnhardt (2004).

Spot 2 is the biggest of the three spots. It is most likely composed of a very large amount of high-order fragments that have been produced by a chain of fragmentation processes (Sekanina et al. 1998; Boehnhardt 2004). Spot2 is $896 \mathrm{~km}$ away from the parent body (Fig. 3) and should contain sub-nuclei that were released earlier than those in Spot1b. We would therefore expect it to show less activity than Spot1b because of dissipation processes. However it has the highest contribution, $\sim 70 \%$, of the gas and dust production, suggesting that all the fragments in the FOV of S-Cam3 might have been created only a few days before the observations. Consequently they still are comparable in activity, and the dust and gas production of each spot is the sum of the activities of all its fragments. As the comet material fills the FOV of the images, the uncertainty in the background level is rather high. However, an error in the background subtraction would mainly affect the intensity of Spot2. Our analysis has, however, shown that, even for this spot, the error in the intensity contribution should not be larger than $\sim 2 \%$ which is derived from the standard deviation of the intensity contributions considering the uncertainty of the background.

\subsection{Temporal variations in dust and gas production in minute levels}

\subsubsection{Intensity variation}

We have investigated the variation of the spatial brightness distribution of Spot 1 and Spot 2 in two-minute time steps. This was done by determining the shift, $(\Delta x \Delta y)$, of the brightness $B_{\mathrm{i}}$ of $n$ images $(i=0 \ldots n)$ with respect to one reference image, $B_{\text {ref }}$, via cross correlation (see Eq. (2)). A mean image, $\bar{B}$ (see Eq. (3)) was created of the shifted images and subtracted from the images $B_{i}$ (see Eq. (4)).

$\Delta x \Delta y=B_{\text {ref }} \bigotimes B_{\text {i }}$

$\bar{B}=\frac{\sum \operatorname{shift}\left(B_{\mathrm{i}}, \Delta x, \Delta y\right)}{n}$

$B_{\text {new }}=B_{i}-\bar{B}$.

This procedure was applied to Spot1 and Spot2. To obtain $B_{i}$ for each spot, we created a median image of seven consecutive images with a $10 \mathrm{~s}$ integration time. This allowed the removal of a star trail $(V=15.3)$ crossing the field of view in the Spot 2 data set, but prevents detection of any intensity variations shorter than 70 s. The intensity variations of Spot 1 and Spot 2 are shown in
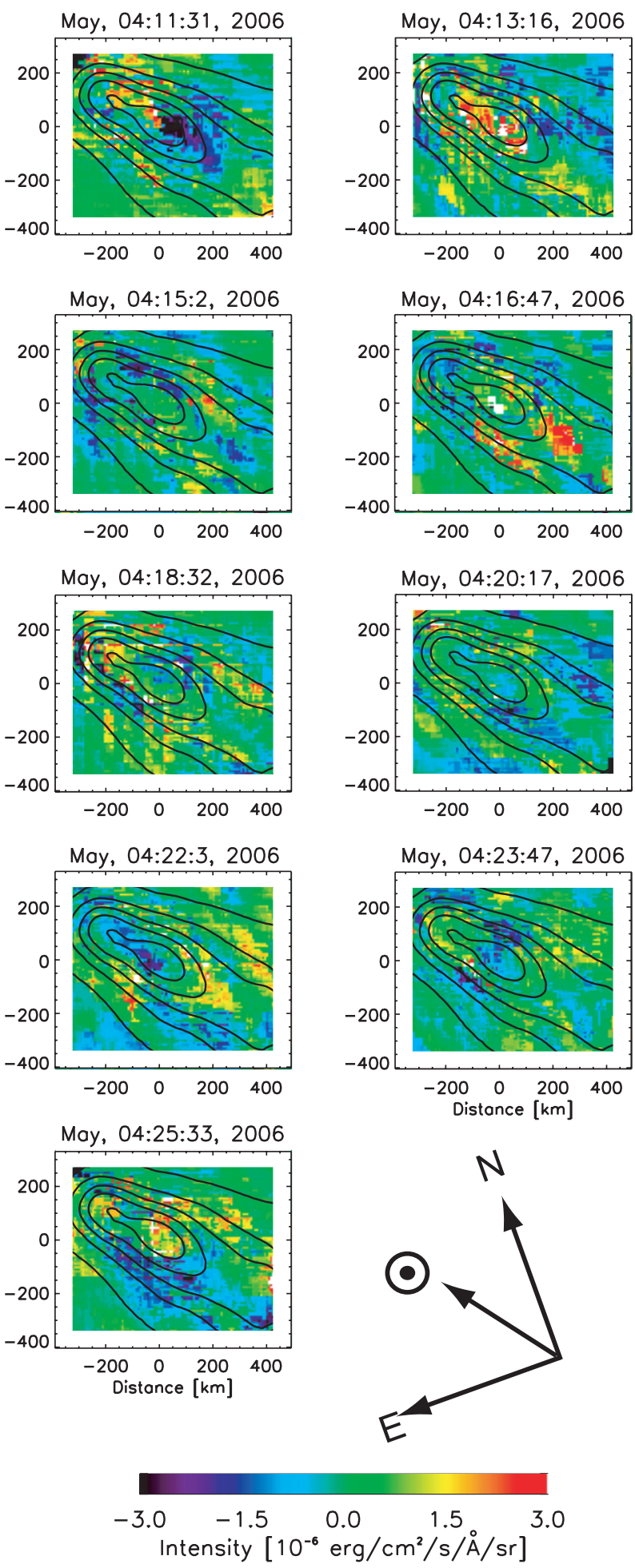

Fig. 4. Brightness variation of Spotla \& b in units of $10^{-6} \mathrm{erg} / \mathrm{cm}^{2} / \mathrm{s} / \AA / \mathrm{sr}$. The subtracted mean image, $B_{\text {ref }}$, is over plotted as contour to facilitate the recognition of the intensity variations. The $(0,0)$ origin of the images is defined by the centre of Spot $1 \mathrm{~b}$ of the $B_{\text {ref }}$.

Figs. 4 and 5, respectively. The pixels at the edges of the frames had to be omitted due to artefacts created by the shifting and were removed to avoid confusion in the analysis.

Both spots show areas of higher and lower intensities. The strong intensity increase of Spot 2 at 04:36:10 UT $(x / y=$ $-250 /-180 \mathrm{~km})$ and 04:37:20 UT $(x / y=200 /-50 \mathrm{~km})$ is the 

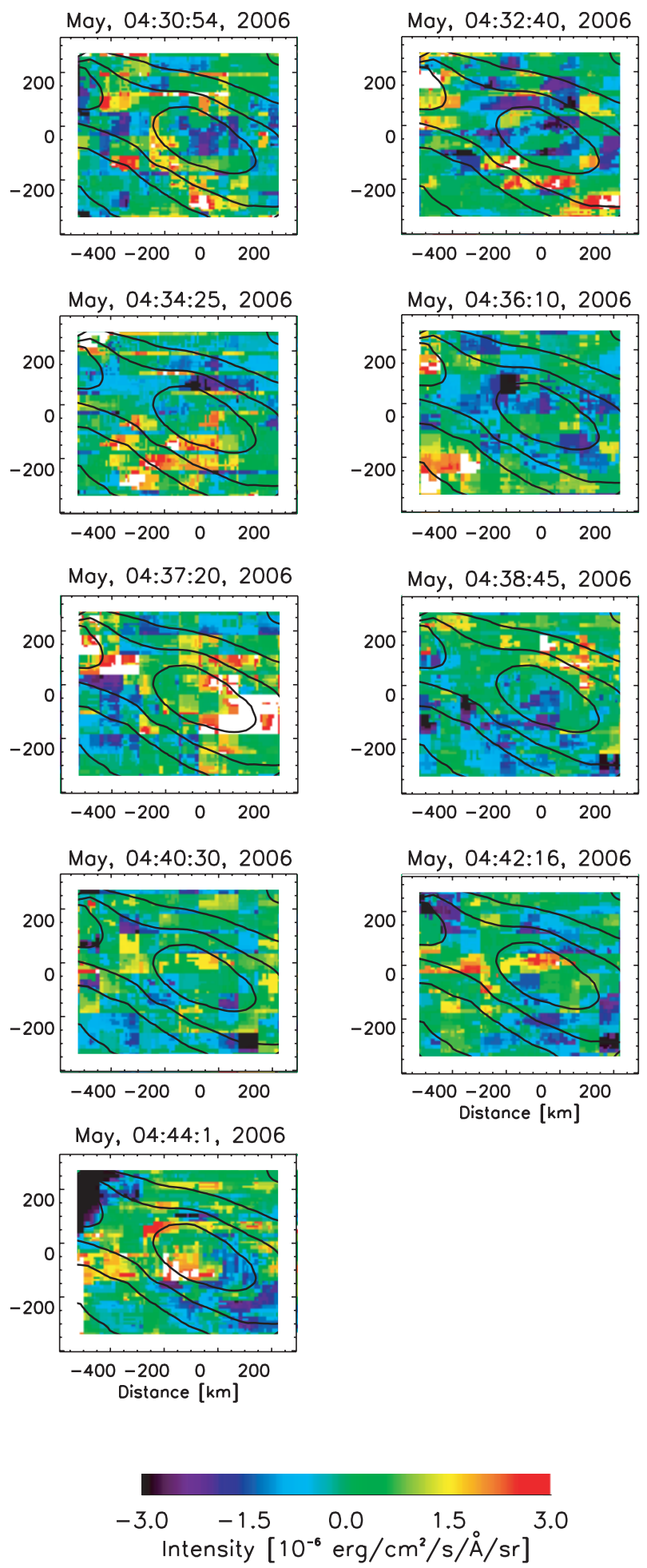

Fig. 5. Brightness variation of Spot2 in units of $10^{-6} \mathrm{erg} / \mathrm{cm}^{2} / \mathrm{s} / \AA / \mathrm{sr}$. The subtracted mean image, $B_{\text {ref }}$, is over plotted as contour to facilitate the recognition of the intensity variations. The $(0,0)$ origin of the images is defined by the centre of Spot 2 of the $B_{\text {ref }}$. North, East and Sun directions are indicated in Fig. 4.

residual of this star passing through the frame during the observation, which could not be removed completely through the median technique. As a result we omitted these features in our interpretation.
We could not identify any structures that are constant on a timescale of $\Delta t>2 \mathrm{~min}$, nor did we detect any periodical effects in these structures. Several intensity increases/decreases $(\Delta I \leq$ 1.5 and $\Delta I \geq 1.5 \times 10^{-6} \mathrm{erg} \mathrm{cm}^{-2} \mathrm{~s}^{-1} \AA^{-1} \mathrm{sr}^{-1}$ ) can be identified in the images indicating lower/higher abundances of gas and dust with respect to the ambient coma. We note that any intensity change $\Delta I$ of $\pm 3 \times 10^{-6} \mathrm{erg} \mathrm{cm}^{-2} \mathrm{~s}^{-1} \AA^{-1} \mathrm{sr}^{-1}$ and of small spatial sizes $\left(<37 \mathrm{~km}^{2}\right)$ might not be real, but they could result from oversampling the image arrays combined with the uncertainties in the shifting (Eq. (2)).

The intensity peaks are not only concentrated in the central regions of Spot1 and Spot2 which contains the main B-body and the highest accumulation of its subnuclei and major fragments, but are scattered around them, see for example Fig. 4 (UT 04:11:31 and UT 04:16:47). A broad distribution of the intensity peaks is seen especially in the images of Spot2 (UT 04:30:54 and UT 04:32:40), see Fig. 5. This and its larger distance to Spot1a indicate that the subnuclei of Spot2 split earlier than the one of Spot1b. Nevertheless both spots show comparable intensity differences.

By taking the ambient intensity of the coma in these regions into account, we obtained a intensity change of $\pm 5 \%$ within $\sim 4 \mathrm{~min}$, which is too low to be attributed to cometary outbursts or further splitting of the subnuclei. (Sub)nucleus splitting should lead to typical brightness changes of about 2 mag and have a duration of a couple of days (Hughes 1990). Activity outbursts usually last several hours. The shortest, with about a one-hour duration, was detected in comet 9P/Tempel (A'Hearn et al. 2005) and is assumed to have been triggered by insulation of active areas. For a comet comparable to comet 73P/SW 3 approaching the Sun at around $1 \mathrm{AU}$, the dust velocity of micron size particles is $\sim 250 \mathrm{~m} / \mathrm{s}$ and the gas velocity is $\sim 700 \mathrm{~m} / \mathrm{s}$ (Gombosi et al. 1986). This is equivalent to $\sim 0.5$ pixel per minute. Consequently, both gas and dust components could not have traveled outside the FOV within two minutes, and we should observe a sharp increase and a gradual decrease in the lightcurve until the gas and dust is out of the FOV or destroyed. Figure 6 presents the total lightcurve (gas and dust) of Spot 1 and Spot 2 integrated over a fixed aperture. The mentioned behaviour cannot be observed for the time span of our data set. It is therefore more likely that the "short" intensity variations detected in our data of 73P/SW 3 result from the varying gas and dust production of subnuclei that are scattered up to distances of $300 \mathrm{~km}$ from the central region with Spot 2 showing a wider distribution of subnuclei than Spot1.

Another possible explanation of these intensity peaks is dust grain fragmentation. In the innermost coma of comets and up to several hundred $\mathrm{km}$ (just outside the dusty-gas dynamic acceleration region), fragmentation can play a major role shaping the intensity profile. It has been suggested previously that thermal stress and dust acceleration trigger dust fragmentation in that region (Thomas \& Keller 1990; Combi 1994). Also the observed heterogeneity of the particle number density in the coma of $81 \mathrm{P} /$ Wild 2 was attributed to fragmentation occurring as a result of enhanced heating, abrupt depressurization, phase transition, and many other possible processes (Clark et al. 2004), most of which are based on the general idea that the matrix material is weakened, leading to disintegration. An increase and a slow decrease in the lightcurve should then be observed as well.

The lightcurve of the dust component is also plotted in Fig. 6 for both spots. The determination of the continuum and thus the dust contribution will be explained in the following Sect. 3.1.2. We observe for Spot1 an increase in the intensity within 2 min 

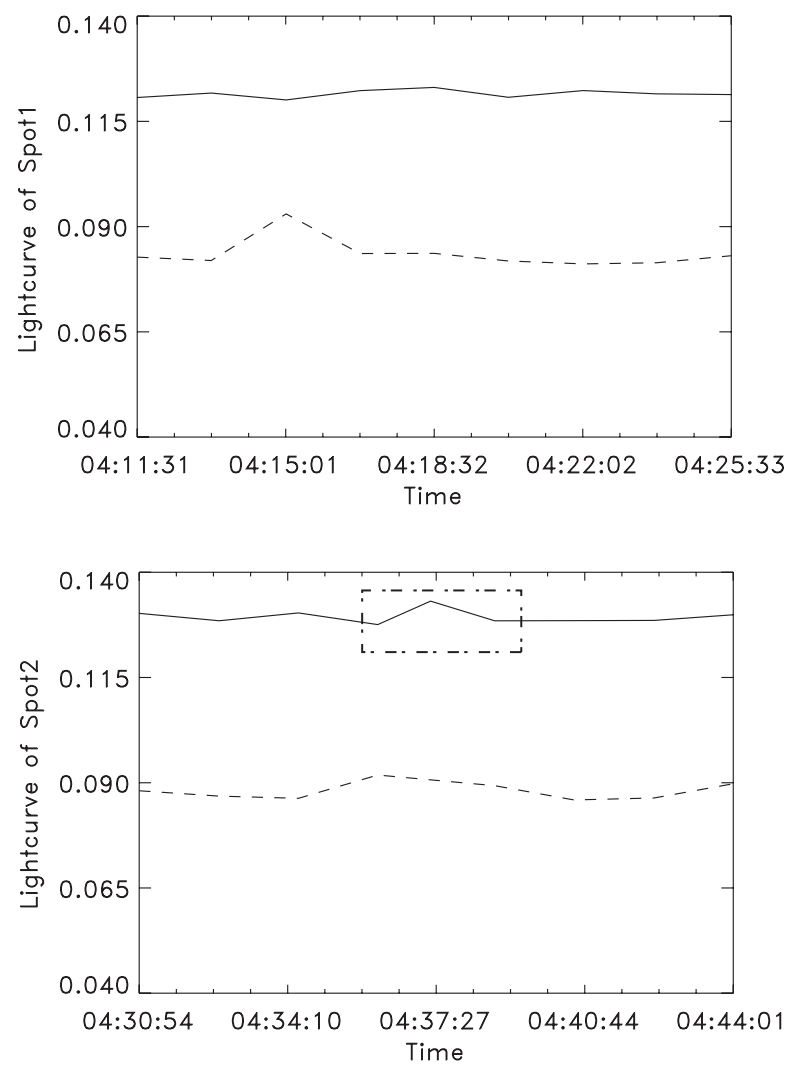

Fig. 6. The total lightcurves (solid line) and the lightcurve of the dust component (dashed line) for Spot1 (upper plot) and Spot2 (lower plot). The box (dashed line) indicates the star passing through the field of view which could neither be removed completely in the total lightcurve nor the dust.

with a decrease of 4 min indicating a rising and sinking amount of dust possibly due to fragmentation or sublimation. The matrix of the dust particles must be so weak that they fragment or dissolve within such a short time scale.

Particles that could be responsible for short time intensity variability might be icy grains. While pure water ice grains have a rather long lifetime even at $1 \mathrm{AU}$, the presence of a very small amount of absorbing material can reduce the lifetime of the particles to minutes or hours (Hanner 1981). If the sublimation of grains embedded with ice is responsible of the short timescale changes, a change in colour towards the blue regime should be observed.

Furthermore, we could observe that the intensity variation of the dust component and the total variation (gas+dust) do not correlate. The evident increase/decrease in Spot1's dust lightcurve goes along with a small decrease/increase in the total intensity; see Fig. 6, most likely indicating the gas contribution. Whether this enhanced presence of gas is directly linked to the drop-off of the dust component is difficult to decide. Because of the uncertainty of fitting a solar spectrum to a S-Cam3 spectra, we were unable to provide a definite conclusion on the contribution of the gas component.

A further source that could lead to the observed variability is the presence of faint background stars. We examined the path traced by the S-Cam 3 detector as it tracked Spot 1 and 2 of fragment $B$ with a view to assessing possible contamination from background field stars in a typical integration time of $2 \mathrm{~min}$. We facilitated the Digital Sky Survey-2 to thoroughly identify any stars that might have crossed the S-Scam3 FOV. The magnitude of such stars would have to be at least 18 to cause variations in the order of those discussed here. Our search revealed stars of magnitude 20 or less.

As described above only one star of visual magnitude 15.3 entered the field of view during the monitoring, and its contribution and contamination has been discussed. In addition, a field of $3^{\prime} \times 3^{\prime}$ was searched for background stars to a limiting visual magnitude of 18 that could possibly contribute to the observed variability through light leakage onto the array from the wings of the telescope point spread function. Only 1 star with a visual magnitude of 14.6 lying some 6" outside the S-Cam field of view could possibly contribute, but only at a totally negligible level given that the seeing was $1.9^{\prime \prime}$ FWHM. Thus background stars can definitely be ruled out as the source for the observed flux variations in 73P/SW 3B.

\subsubsection{Colour variation}

It is well known that comets show significant variations in continuum colours. As these differences are not correlated with heliocentric distance, phase angle, or mass-loss rate, they should result from intrinsic differences in the dust properties of different comets such as grain size distribution and refractive index (e.g. Jewitt \& Meech 1986). Analysing the dust colour of a disintegrating comet can therefore shed some light on the question of whether different dust properties are related to the part of the nucleus from which the dust particles have been ejected. The colour of the dust depends on the grain size, the grain shape, and the optical constants of the material forming the dust particles. Grains that are small compared to the wavelength satisfy the Rayleigh approximation. Thus the scattered radiation is relatively blue. For larger grains, the scattering efficiency falls from the maximum towards the large-particle limit, and scattered radiation in this regime is relatively red (Jewitt \& Meech 1986). Thus reddening is indicated by $S^{\prime}\left(\lambda_{1}, \lambda_{2}\right)>0$ and blue scattered radiation by $S^{\prime}\left(\lambda_{1}, \lambda_{2}\right)<0$.

The colour of cometary dust is proportional to the normalized reflectivity gradient (Jewitt \& Meech 1986; A'Hearn et al. 1984) and is expressed in \%/1000 $\AA$. This reddening percentage can be obtained from following equation:

$S^{\prime}\left(\lambda_{B}, \lambda_{R}\right)=\frac{I_{B}-I_{R}}{\lambda_{B}-\lambda_{R}} \times \frac{2000}{I_{B}+I_{R}}$

where $I_{R}$ and $I_{B}$ are the intensities (relative to the Sun) at the wavelength, $\lambda_{R}$ and $\lambda_{B}$ given in $\AA$.

To obtain colour information, we extracted the blue and red continua at 4453 and $6780 \AA$ (width of $71 \AA$ ) by choosing the corresponding PHA range (see Eq. (1)). We have created blue and red images (I445 and I678) for each data set from which colour maps of the dust continuum were produced. These wavelength ranges were chosen after considering the limited spectral resolution of the S-Cam3 and the signal strength but also taking into account that they should be free of gas (Farnham et al. 2000).

Figure 7 presents two spectra of two selected regions to illustrate the performance of S-Cam3. Because of the instrument's limited resolution the selected continuum images might possibly be contaminated by certain gas species. Since we were not able to provide any definite conclusion of the gas component of the S-Cam3 data, we have used the observational results of comet 73P/SW-3B obtained by Schleicher et al. (2006) on April 25, May 9 and May 10, 2006 to obtain the gaseous impurities. Possible gas specious which could contribute to the blue and red continua are $\mathrm{CN}, \mathrm{C}_{3}$ and $\mathrm{C}_{2}$. Species such as $\mathrm{CO}^{+}$and 


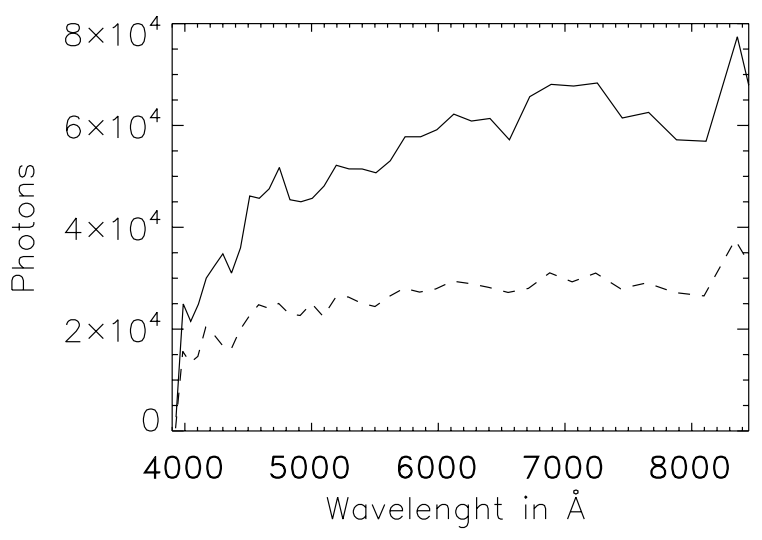

Fig. 7. Spectra of the two selected regions (upper box = solid line \& lower box $=$ dashed line) which are presented in Fig. 2. They are extinction and instrumental response corrected.

$\mathrm{H}_{2} \mathrm{O}^{+}$are of minor importance due to their low production rates and therefore can be ignored.

From the $\mathrm{CN} / \mathrm{Af} \rho, \mathrm{C}_{3} / \mathrm{Af} \rho$ and $\mathrm{C}_{2} / \mathrm{Af} \rho$ ratios, we were able to calculate the theoretically observed intensities and thus obtained a gas contamination between $18-23 \%$ for the blue continuum. Most is $\mathrm{C}_{3}$. The amount of gas influencing the red continuum is negligible. If we remove $20 \%$ of the measured intensity of the blue continuum, we would redden the resulting colours by $+9 \%$ assuming a constant dust to $\left(\mathrm{CN}, \mathrm{C}_{3}, \mathrm{C}_{2}\right)$ gas ratio.

The representative colour maps for Spot 1 and Spot 2 in percentage reddening are presented in Figs. 8 and 9. The colour maps still include the gas contribution since there is an uncertainty in the dust to gas ratio for the night of the S-Cam3 observation. Bertini et al. (2007) have reported outbursts on April 25 and May 8, 2006 for fragment B indicating its high activity within the time frame covering the S-Cam3 observations.

The mean dust colour of Spot 1 and Spot 2 varies every two minutes by $\pm 2 \% / 1000 \AA$ within an interval of [4453 $\AA, 6780 \AA$ ]. However we observed a significant decrease of $\sim 10 \% / 1000 \AA$ of the mean colour at 04:15:02 UT for Spot1. This coincides with a brightness increase within $2 \mathrm{~min}$ in the dust contribution presented in Fig. 6. Afterwards a reddening follows combined with a drop-off of the intensity over $5 \mathrm{~min}$.

Sublimation of icy grains would give a blue reflectivity gradient and a steepening brightness as observed by Lara et al. (2001) for comet C/1996 Q1 Tabur; therefore, the intensity variation of Spot1 could instead indicate an increased amount of smaller particles possibly because of fragmentation or extended emission of gas and smaller dust grains. A relative colour gradient of $-10 \% / 1000 \AA$ suggests a noticeable change in the particle size distribution of the ejected grains if the total intensity increase can be assigned to the dust component of the coma only. This increase/decrease have been observed in both continua. We observe in contrast a weak brightness decrease/increase in the total lightcurve at same time, see Fig. 6, indicating the variation of the gas component. Since the blue continuum has a non negligible gaseous leakage no definite conclusion of the gas and dust contribution, responsible for the observed intensity and colour changes, can be made. However, the different behaviours of the total and the dust lightcurves suggest that the brightness increase/decrease in the blue continuum is most likely of dusty origin.

We did not obtain any significant colour and lightcurve changes for Spot2. The dust lightcurve of Spot2 shows less variation, so the colour suggests a more or less constant size
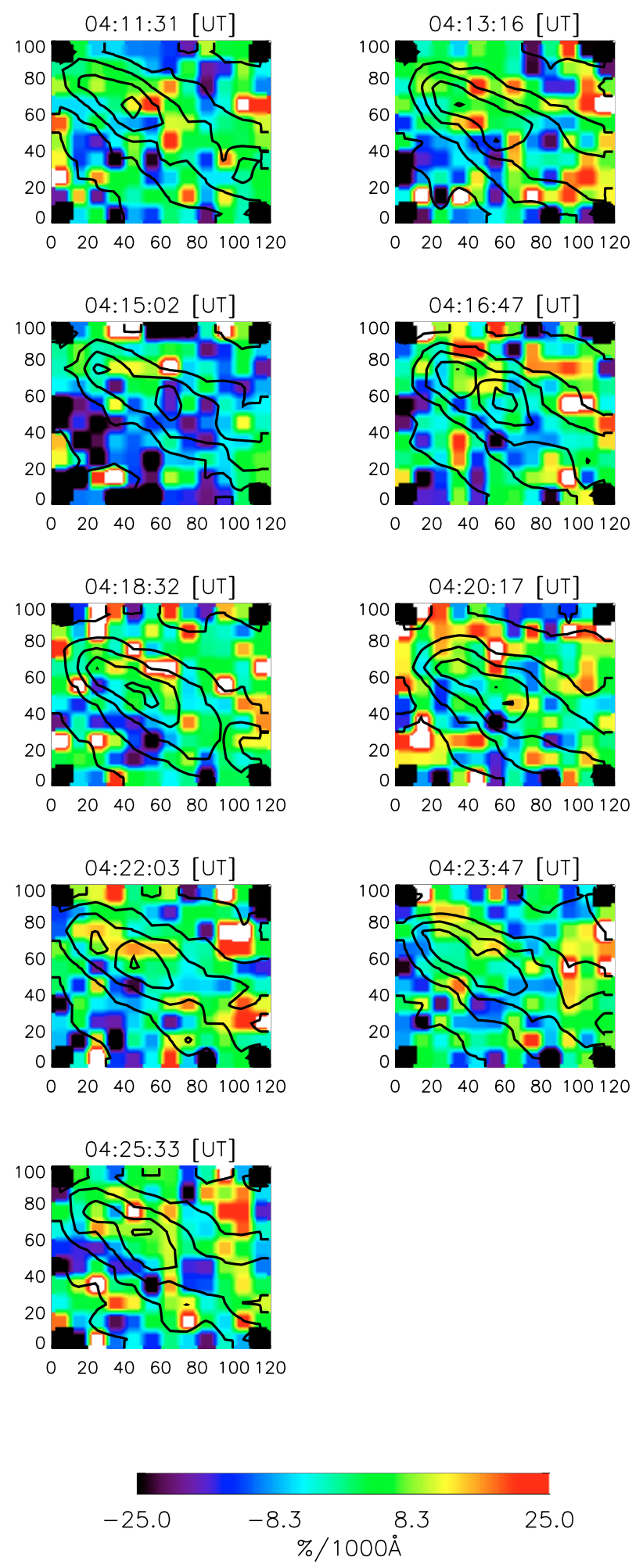

Fig. 8. Colour variation of Spot 1 in $\% / 1000 \AA$. Each pair of red and blue image has been added and overplotted as contour for a better outline. North, East and Sun direction are indicated in Fig. 4.

distribution. The colour uncertainty due to the $\mathrm{S} / \mathrm{N}$ ratios is negligible.

\subsection{Radial profiles}

The dust grains emitted from a comet nucleus are coupled in the region close to the nucleus surface (gas-dust interaction region) 

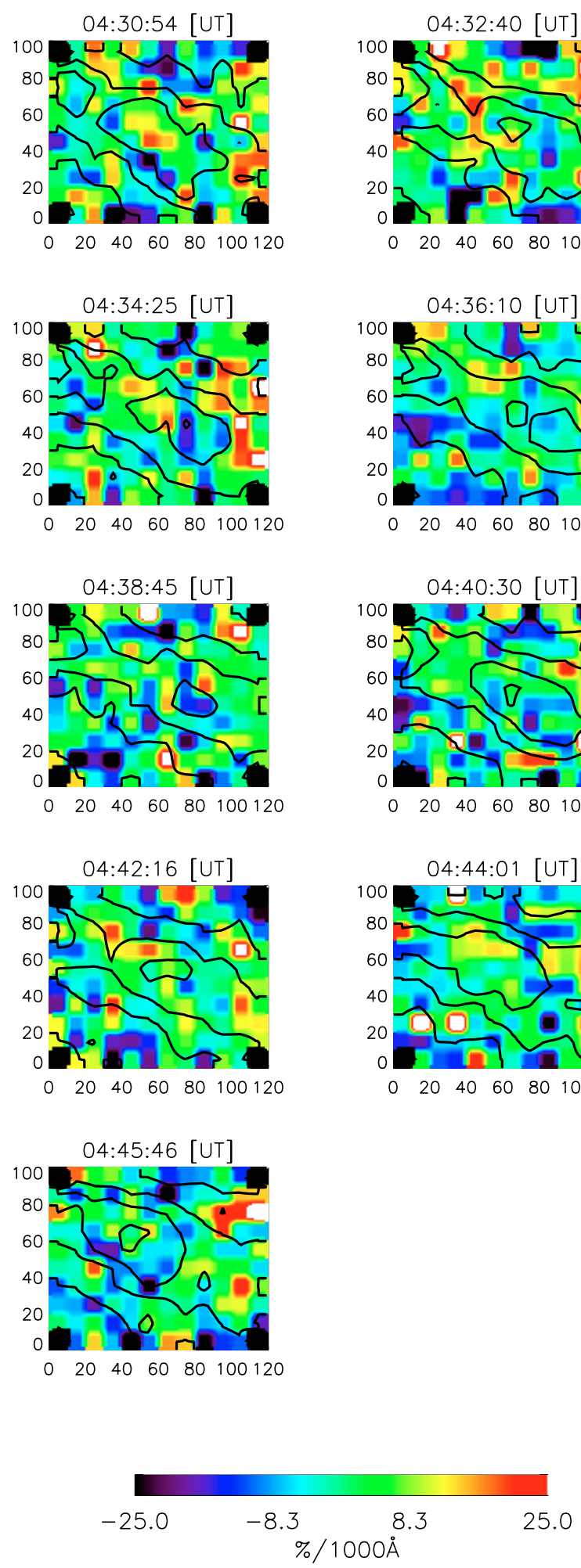

Fig. 9. Colour variation of Spot 2 in \%/1000 A. Each pair of red and blue image has been added and overplotted as contour for a better outline. North, East and Sun direction are indicated in Fig. 4.

more or less strongly with the gas, depending on their sizes. Thus their motion is described by an acceleration region and an asymptotic approach to their terminal velocity within a few tens of nuclear radii (Gombosi et al. 1986). At distances above $300 \mathrm{~km}$, the dust particles finally reach force-free radial outflow, also called $1 / r$ behaviour. Beyond a distance from the nucleus of
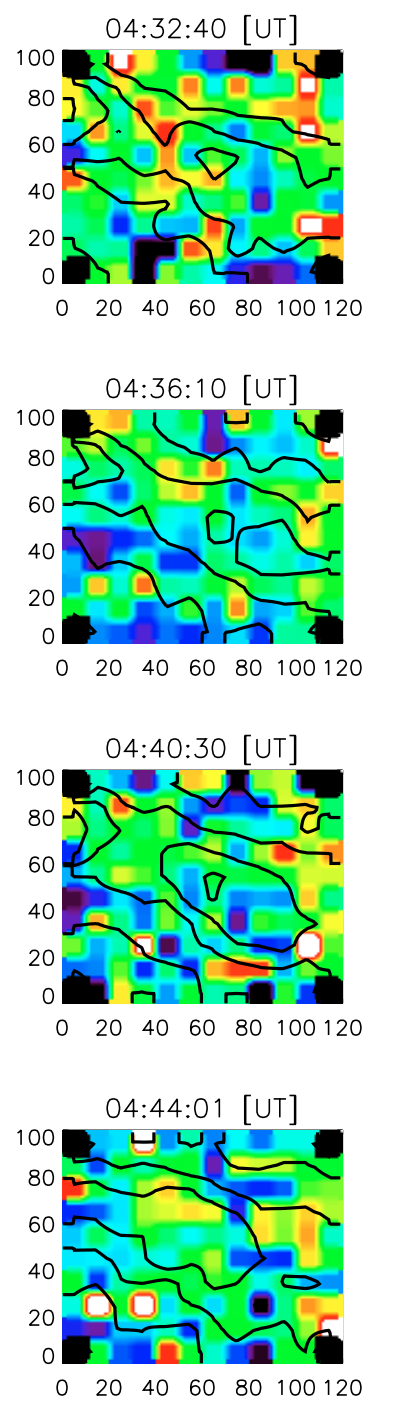

T. M. Ho et al.: The coma of 73P/Schwassmann-Wachmann 3-B

a few times $1000 \mathrm{~km}$, the particles are increasingly influenced by solar radiation pressure forces.

With a FOV of $1241 \mathrm{~km} \times 949 \mathrm{~km}$ of the S-Cam 3 images and a pixel scale of $73 \mathrm{~km}$, we are able to examine the dust outflow in the first few hundred kilometres away from the emission sources by investigating any trend in dust and gas outflow with aperture size (such as deviation from free radial outflow). However, we have to bear in mind that we do not have one single nucleus at the centre of the coma in the case of comet 73P/SW 3-B, but several active "mini-comets" forming an extended source region. The determination of the coma centre, and the radial profile originating from it, is not straightforward. For our data analysis we used the region of maximum intensity as the centre of each image.

We investigated the dust and gas flow of Spot1b and Spot2. The four radial directions that are significant for our analysis are depicted in Fig. 10. They represent (1) the sunward direction, (2) the tail direction and the perpendicular cuts defining the distribution in northern, (3) and (4), southern direction. Because of the very small distance between Spotla and Spot1b and the low spatial resolution, an investigation of the gas and dust flow between these two fragments was impossible, and the corresponding profile was therefore omitted in our analysis.

Figures 11 and 12 show the radial profiles of Spot $1 b$ and Spot 2 in double logarithmic representation, taking the images at $4453 \AA$ as an example. To improve the signal and spatial resolution, the normalized images of Spot 1 and Spot 2 were shifted according to Eq. (3) and stacked. In addition a median filtering was performed to remove the star track. For the images of the whole bandwidth (RX), 395-1052 nm, containing the signal of the dust and the gas contribution, we also plotted the radial profiles to acquire evidence of the gas outflow.

The dust radial profiles of Spot1b are slightly steeper and smoother than those of Spot2, which may be explained by a closer grouping of sub-nuclei within Spot1b. Each sub-nucleus emits gas and dust, influencing the emission of their immediate neighbours and, resulting in a "disturbed" outflow. Hence the outflow of a closely grouped cluster of sub-nuclei follows a $1 / r$ profile. In addition, as the fragments are trailing behind each other, we would expect the profile in the tail direction to contain 


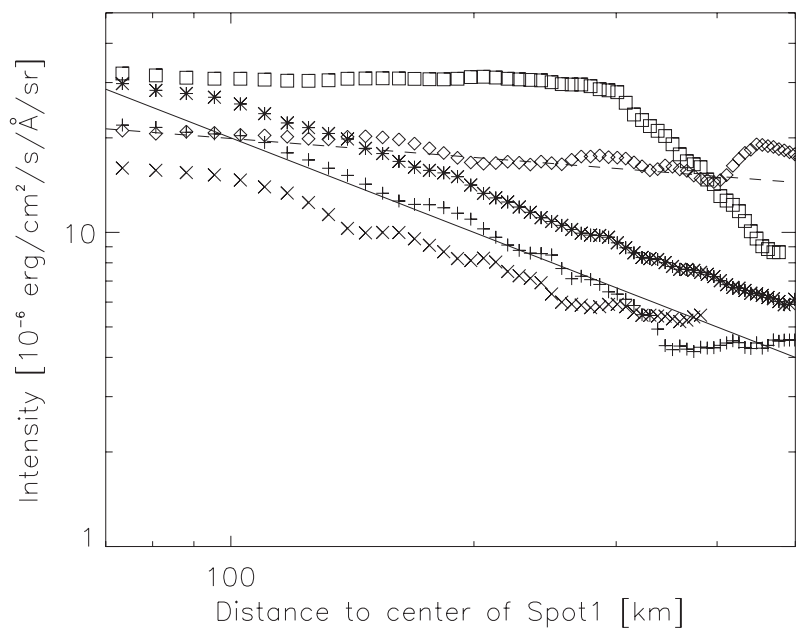

Fig. 11. Radial profiles of Spot $1 b$ shown in double logarithmic representation. For the dust profiles the reflectivity at $4453 \AA$ is given as a function of the projected distance, $\rho$ to the centre. $(\diamond)$ : tailward direction, $(+)$ : southern direction, $(\times)$ : northern direction. The sunward direction is not plotted, because of the small distance to Spotla. The profiles integrated over the entire wavelength range reflecting a mixture of gas and dust (RX) are shown in tailward ( $\square$ ) and southern $(*)$ direction.

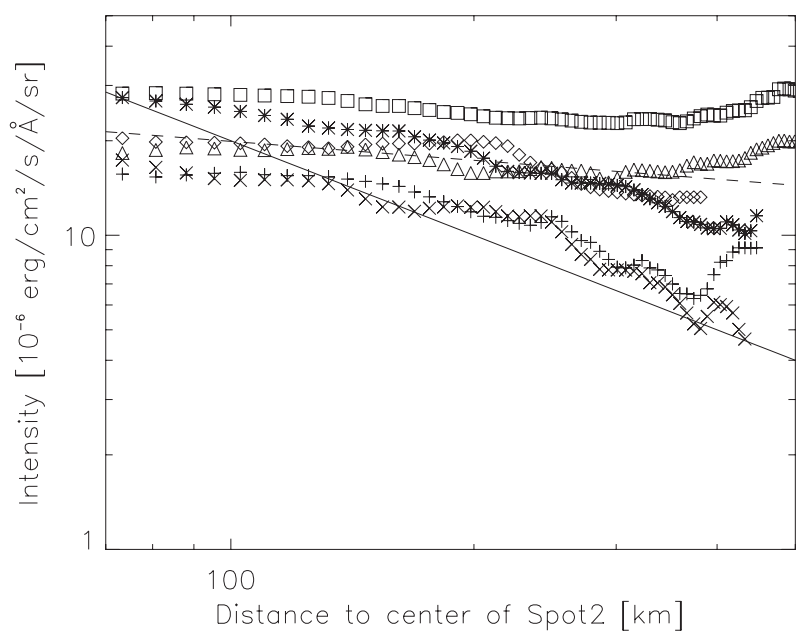

Fig. 12. Radial profiles of Spot 2 shown in double logarithmic representation. For the dust profiles the reflectivity at $4453 \AA$ is given as a function of the projected distance, $\rho$ to the centre. $(\triangle)$ : Sunward direction, $(\diamond)$ : tailward direction, $(+)$ : southern direction, $(\times)$ : northern direction. The profiles integrated over the entire wavelength range reflecting a mixture of gas and dust (RX) are shown in tailward ( $\square$ ) and southern $(*)$ direction.

more gas and dust sources and therefore should be less smooth compared to the perpendicular ones.

For both Spot1b and Spot 2 the profiles in perpendicular direction decrease as $1 / r$, whereas those along the sun-comet line have much flatter gradients. This clearly indicates that other effects influence the profiles in the direction of the sun, as well as of the tail. A possible explanation could be the "downward stream" outflow of Spot1b meeting the "upward stream" of Spot2. The gas and dust outflow of both spots would mix and result in an apparently flatter radial profile compared to outflows that are not influenced by any opposite ones (as in the case of the perpendicular emission). Of course differential atmospheric refraction could also influence the radial profiles; however, the

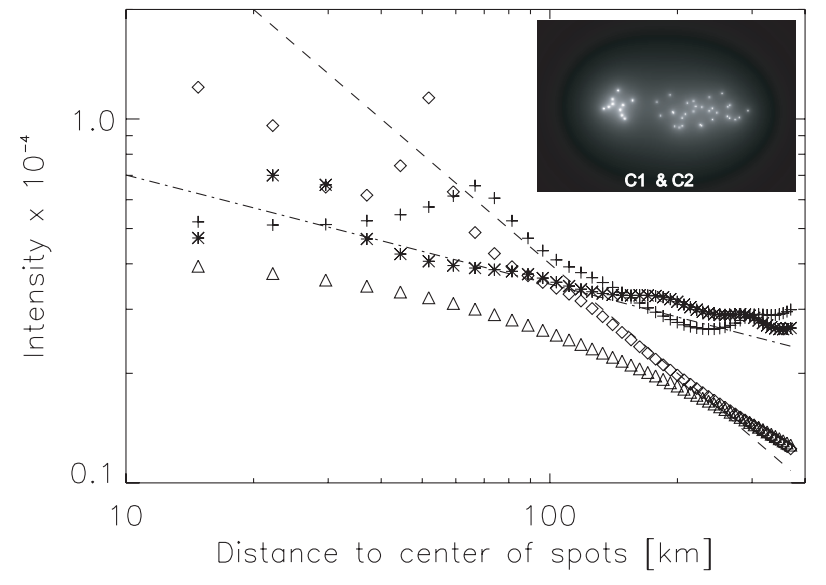

Fig. 13. The profiles of two clusters of subnuclei. The image is presented in the upper right corner. $\mathrm{C} 1$ has 10 subnuclei and $\mathrm{C} 2$ has 30 subnuclei. The radial profiles which correspond to the profiles of Fig. 10 are (1) the profile along the Spot1-Spot2 line $(+),(2)$ its normal profile $(\diamond)$, (3) the profile along Spot2-Spot1 (*) and (4) its normal $(\triangle)$. The dash line with a slope of -1 represents the force-free radial outflow and the dash-dot line a $1 / r^{-0.3}$ outflow.

observations were conducted at an airmass $\approx 1$ thus there is no effect of differential atmospheric refraction on our analysis.

Another explanation could be fragmentation processes of bigger dust grains into micron and submicron particles that are optically larger in the visible wavelengths. Hence the observed column density does not increase. To investigate this scenario, we simulated two clusters of sub-nuclei representing Spot $1 \mathrm{~b}$ and Spot2, which isotropically emit dust and gas with constant velocities. Thus we can create a coma of multiple point sources with a $1 / r$ fall-off. This is of course a very simple model that excludes influences, such as the acceleration, radiation pressure, possible interaction between colliding opposite dust and gas outflow, and fragmentation processes. The zone in which the dust decouples from the gas starts at distances $>300 \mathrm{~km}$. However, the decoupling length also depends on the particle sizes, whereby smaller particles are embedded for longer within the gas flow. Furthermore, the gas and dust particles almost reach their terminal velocities at a few tens of the nuclear radius, therefore acceleration might not be the most dominant characteristic observed in the radial profiles within this region. As we examine the outflow within the first $500 \mathrm{~km}$ away from the emission centres, radiation pressure is not the dominant force and thus free radial outflow remains valid. Figure 13 presents the modelled radial profiles of two clusters of sub-nuclei. The first cluster (C1) contains 10 normally distributed sub-nuclei and the second cluster (C2) contains 30 sub-nuclei. The positions are determined randomly. The Gaussian distributions of source positions have standard deviations that result in equivalent FWHM of Spot $1 \mathrm{~b}$ and Spot2. The two clusters are positioned $643 \mathrm{~km}$ away from each other.

Our model calculations result in radial profiles that are significantly flatter between the clusters than in the perpendicular direction. There are two peaks observed in the radial profiles. These are point sources that are located along the profile line. The perpendicular profiles of both clusters are steeper, but they do not follow the $1 / r$ fall-off of the observations. The perpendicular profile of $\mathrm{C} 1$ follows the $1 / r$ more than the one of $\mathrm{C} 2$ because the sources are positioned closer.

We have further investigated the radial profiles for changes. The tailward profile's gradient can vary between -0.2 and -0.3 , 
and the profile of the normal between -0.8 and -1 every two minutes. For Spot 2 we obtain gradients for the perpendicular direction between -0.6 and -0.7 .

\section{Summary and conclusion}

The S-Cam3 observations of comet 73P/SchwassmannWachmann 3-B show three clusters of fragments in the gas and dust coma that have been divided into Spot1a, b and Spot2. Due to its leading position and its equal dimension of $131 \mathrm{~km} \times 134 \mathrm{~km}$, Spotla most likely contains only the main B-body or very few additional fragments that were just created and still in the immediate vicinity of the parent body. Spot $1 \mathrm{~b}$, however, has a more elongated shape $(177 \mathrm{~km} \times 302 \mathrm{~km})$ containing a higher number of sub-nuclei produced through further fragmentation processes. These are occurring mainly in the tail direction and are more dispersed. The third intensity maximum forms Spot2, which has the largest dimension $(334 \mathrm{~km} \times 825 \mathrm{~km})$ and suggests that more break-ups have occurred. As the fragments forming Spot2 are products of multiple fragmentations, we would expect them to be smaller pieces. This should lead to lower activity and/or dissipation processes. However, Spot2 has the highest dust and gas production of all three spots. Our investigation does not show evidence for significant differences in the intensity variation of Spot1 and Spot2 on small time scales, suggesting that both spots were formed in a short time span (a few days) and contain relatively large fragments with comparable activities. Both spots show comparable spatial intensity variations $(\approx 5 \%)$, most likely indicating dust and gas production rates of individual sub-nuclei.

Because of the low spectral resolution of the S-Cam3 instrument, we were not able to obtain a blue continuum which is free of gas. The simulations of the possible gas contamination yield that $\sim 20 \%$ of the observed blue continuum signals could be gaseous indicating that the actual dust colours might be redder than the results presented in Figs. 8 and 9.

The mean dust colours of Spot1 and Spot2 vary between $\pm 2 \% / 1000$ Å reddening suggesting most likely a minor change of the particle size distribution assuming a constant $\mathrm{CN}, \mathrm{C}_{3}$, $\mathrm{C}_{2}$ to dust ratio. However, we were able to detect a significant change in colour within $6 \mathrm{~min}$ in Spot1, which goes along with an increasing/decreasing integrated dust intensity. This could indicate an enhanced emission of small particles or dust fragmentation. Because of the gas leakage of the blue continuum no definite conclusion can be derived whether the dusty or gaseous part is mainly responsible for the intensity and colour changes. However, the simultaneously observed opposite behaviour of the total lightcurve suggest that the dust component is most likely responsible for the changes in Spot1. The lightcurve does not show the expected sharp increase and slow decrease characteristics, suggesting this process has happened on a small scale, rather than an intense activity such as an outbursts. No similar behaviours could be seen in Spot2 within its observational time frame.

The dust and gas outflow between the spots do change the appearance of the overall gas and dust flow. We basically observed a difference between the radial profile in the Sun-comet line and the one in a perpendicular direction. The former is composed of two outflows in opposite directions and the latter shows a pattern consistent with one outflow direction. The observations could be reproduced in principle by a simple outgassing model that assumes multiple point sources although the modelled perpendicular profile is somewhat flatter than what is observed. As our model is additive, we have not considered any physical processes that might have happened when two opposite dust and gas flows collide. Another scenario that could flatten the profiles is fragmentation of dust grains into particles, which are optically more active in the visible wavelength range. The dust images show flatter sunward-tailward profiles; however, their perpendicular profiles are steeper and approaches the $1 / r$ fall-off, which would indicate that any fragmentation processes occur mainly along the Sun-comet line.

The observations describe the first hundred $\mathrm{km}$ of comet 73P/SW 3B with dust and gas emissions of the subnuclei flowing into each other and steady dust-fragmentation processes. The detection of a small intensity peak in Spot1 indicates a localized enhanced production of small grains, presumably because of fragmentation or varying emissions of the subnuclei suggesting their different activities.

Acknowledgements. We appreciate the referees' recommendations that helped us to improve the paper. We wish to thank D. Schleicher who kindly provided us with the gas and dust production rates and L. M. Lara \& I. Bertini for the outbursts informations of 73P/SW 3B.

\section{References}

A'Hearn, M. F., Schleicher, D. G., Millis, R. L., Feldman, P. D., \& Thompson, D. T. 1984, AJ, 89, 579

A'Hearn, M. F., Belton, M. J. S., Delamere, W. A., et al. 2005, Science, 310, 258 Bertini, I., Boehnhardt, H., Gutierrez, P., et al. 2007, in EPSC Abstracts, 2, 2007A-00044

Boehnhardt, H. 2004, Comets II, 301

Boehnhardt, H., Rainer, N., Birkle, K., \& Schwehm, G. 1999, A\&A, 341, 912

Clark, B. C., Green, S. F., Economou, T. E., et al. 2004, J. Geophys. Res. (Planets), 109, 12

Combi, M. R. 1994, AJ, 108, 304

Crovisier, J., Bockelee-Morvan, D., Gerard, E., et al. 1996, A\&A, 310, L17 Farnham, T. L., Schleicher, D. G., \& A'Hearn, M. F. 2000, Icarus, 147, 180 Gombosi, T. I., Nagy, A. F., \& Cravens, T. E. 1986, Rev. Geophys., 24, 667 Hanner, M. S. 1981, Icarus, 47, 342

Hughes, D. W. 1990, QJRAS, 31, 69

Jewitt, D., \& Meech, K. J. 1986, ApJ, 310, 937

Lara, L. M., Schulz, R., Stüwe, J. A., \& Tozzi, G. P. 2001, Icarus, 150, 124

Martin, D. D. E., Verhoeve, P., Oosterbroek, T., et al. 2006, in Presented at the Society of Photo-Optical Instrumentation Engineers, SPIE Conf., Groundbased and Airborne Instrumentation for Astronomy, ed. Ian McLean, S., Masanori, I., Proc. SPIE, 6269, 626900

Schleicher, D. G., Birch, P. V., \& Bair, A. N. 2006, BAAS, 38, 485

Sekanina, Z. 1982, in Comet Discoveries, Statistics, and Observational Selection, ed. L. L. Wilkening, IAU Colloq., 61, 251

Sekanina, Z. 1999, A\&A, 342, 285

Sekanina, Z., Chodas, P. W., \& Yeomans, D. K. 1998, Planet. Space Sci., 46, 21 Thomas, N., \& Keller, H. U. 1990, Ann. Geophys., 8, 147

Weaver, H. A., Lisse, C. M., Mutchler, M. J., et al. 2006, in AAS/Division for Planetary Sciences Meeting Abstracts, 38, 06.02 